Volume 8, No. 7, July - August 2017

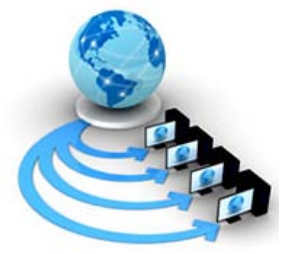

International Journal of Advanced Research in Computer Science

REVIEW ARTICLE

Available Online at www.ijarcs.info

\title{
EYE PHONE-HAND FREE MOBILE CONTROL: A REVIEW
}

\author{
Saba Hilal \\ Student, Computer Science Engineering \\ SSM College of Engineering \& Technology \\ Kashmir, India,
}

\author{
Syed Nazimul Islam Bukhari \\ Student, Computer Science Engineering \\ SSM College of Engineering \& Technology \\ Kashmir, India,
}

\author{
Zubair M Paul \\ Assistant Professor, Computer Science Engineering, \\ SSM College of Engineering \& Technology, \\ Kashmir, India,
}

\begin{abstract}
Eye Phone, a new "hand-free" interfacing system which enables its users in driving mobile applications/functions using only the eyes movement and actions. Using the front camera of the phone and different machine learning algorithms, user's eye movements are being tracked. Eye Phone tracks the position of the user's eye relative to the screen but not where the user is looking. Eye phone seems to be an important alternative to voice activation systems as their performance decreases in noisy environment. The naturalness of gaze makes eye tracking reliable and promising. The Eye Phone’s working mainly relies on eye tracking and blink detection. This paper gives an overview of eye tracking system and prototype implementation of Nokia N810 developed at Dartmouth College.
\end{abstract}

Keywords — Human-Phone Interaction, Machine Learning, Mobile Phones, On Board Sensors.

\section{INTRODUCTION}

As the research on the smart phones is continuously going on, researchers are also trying to ease the human-phone interaction (HPI). After touch sensitive screens to voice activation systems and now to EyePhones that operates on eye tracking software and algorithms will be much interesting and attractive to various users. Voice control is an alternative way to hand free systems but their performance decreases in noisy environments. A team at Dartmouth College has worked on eye tracking and led to the development of eye tracking systems that tracks the user's eye movement for controlling and operating smart phones with eye actions. Eye tracking has been used for years by persons with disabilities and in military applications too. However, the real challenge is to track the eye movement on a small screen like cell phones. The Dartmouth researchers devised an algorithm that identifies a person's eye under different conditions, at varying distances and under different lighting conditions [1].Eye tracking seems to be more challenging on a cell phone as because the eye barely moves when looking to items very close on a screen. Eye Phone is a new "hand-free" interfacing system which enables its users in driving mobile applications/functions using only the eyes movement and actions. Eye phone tracks the user's eye movement on the screen simply by the front camera of the smart phones. Also, different machine learning algorithms are used to track and infer the position of eye on the users viewed screen and to detect the blink that reproduce and generates a function similar to a mouse click. For experimental purpose, the eye phone implementation on Nokia N810 was carried out which actually was the prototype implementation [2]. This implementation was capable of tracking the position of

user's eye and the blink detection. Eye tracking is nothing new however it is very difficult on small screens. Eye tracking at beginning involves a learning phase in which different pictures of eyes are being captured and that too under different conditions of light and surroundings. This information is being stored and when the user views the screen for certain operation, the eye tracking software then divides the camera frame into nine regions and starts looking for the eye in one of those regions [1, 2]. The user has to adjust the camera phone in such a way that a virtual error box is being created around the user's eye. After that only the user can operate the phone functions in a reliable manner. Blinking equates a mouse click that is for selection purpose. Existing algorithms are highly inaccurate in mobile conditions as because a

slight movement can be easily guessed by a signature. This is a basic system and research is still going on perfect and reliable eye tracking algorithms and software's [2].

\section{EYE PHONE DESIGN}

Important things that needs to be taken care of in designing an Eye Phone is how we can use cheap, existing, omnipresent sensors and hardware's in designing of an Eye phone. Also, importantly eye tracking software and blink detection mechanisms are to be carefully designed and algorithms to be used must be accurate and reliable. The Eye Phone algorithm design can be divided into the following pipeline phases: 
1) An eye detection phase;

2) An open eye template creation phase;

3) An eye tracking phase;

1) An eye detection phase:

The first step is to locate the eyes and then analysing the blink of the user [3]. This phase involves finding the contour of the eyes by applying motion analysis technique. The eye detection is very early and quite important phase but hardware and conditions affect that too. When the eye is being detected using a fixed error free camera, the eyes are being properly identified however using Nokia N810 camera a number of errors are detected and there is improper detection of eye contour. So therefore, a proper focus should be on camera design and sensors used [1,3]. The contour pair detects the right and left eye pair accordingly. By reducing the resolution, we can correct the error rate of eye detection and we can add thresholds to filter out the false eye contours.


Left figure: example of eye contour pair returned by the original algorithm running on a desktop with a USB camera. The two white clusters identify the eye pair. Right figure: example of number of contours returned by EyePhone on the Nokia N810. The smaller dots are erroneously interpreted as eye contours.

Fig 1 Eyedetection comparison with fixed USB camera and Nokia N810 camera.

\section{2) An open eye template creation phase:}

The system will track the user's open eye. At this point of time we have to understand that an improper template can be created because of blinking of the user. After that the timer is started and that too when the eye is properly detected [3]. The approximate time needed for the user's eye to become open again after an involuntary blink is detected and then a small number of frames elapse are generated after which the template of the user's open eye is created. The template is saved in the persistent memory of the device so that they can use memory instructions and fetched when an Eye Phone is invoked. Thus, at template creation the user is assumed to be blinking at normal rate [1, 3].

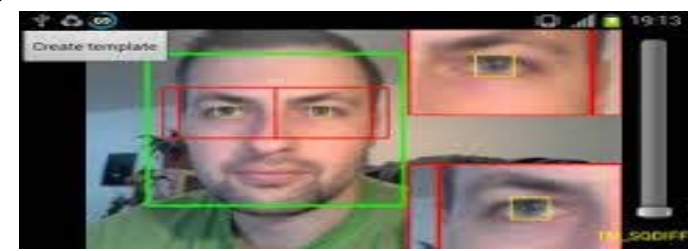

Fig 2 Template creation
No offline templates are necessary and the creation of the online template is independent of any previous template that may have been created. The diversity in the templates varies in size as well as tight around the eye to a larger area surrounding the eye, including the eyebrows.

\section{3) An eye tracking phase:}

Eye tracking is an important tool to study different features of visual interpretations and perception. Eye tracking helps us to better understand the tasks and movements of eyes so that we can better explore the guidance and navigation of visual attention [4]. Template matching is the basis for eye tracking algorithm. Since this method requires an extensive amount of computation and is performed 30 times per second [3]. The area (search area) around the user's eye is also restricted for the computation purpose and thus the system is allowed to run smoothly in real time. The correlation score between the open eye and search window is calculated. This calculation is performed by template matching function. Along with that we have a correlation coefficient that is normalized and ranges between -1 to 1 [1].
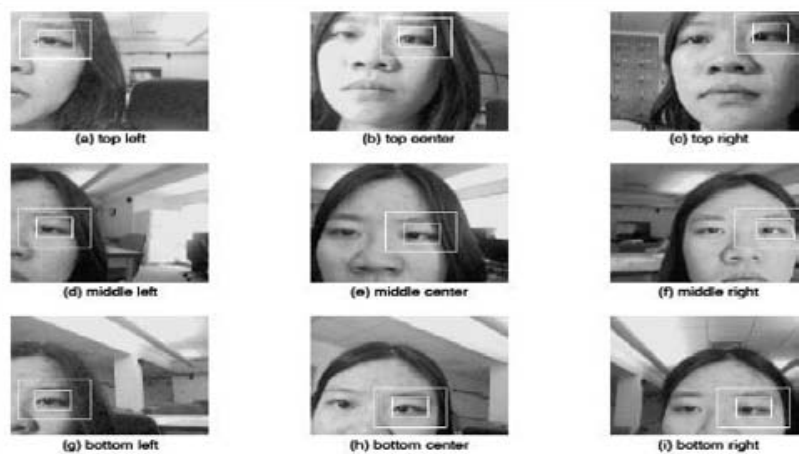

Fig 3Eye captures using the Nokia N810. The inner white box is used to discriminate the nine positions of the eye on the phone's display and the outer box encloses the template matching region [1].

4) A blink detection phase:

The blink duration analysis and blink detection is fully based on correlation scores generated by eye tracking phase. In the algorithm being used four thresholds are being derived in an experiment and the four thresholds are:

$\operatorname{Tmin} 1=0.64, \operatorname{Tmax} 1=0.75$,

Tmin $2=-0.53$, and $\operatorname{Tmax} 2=-0.45$.

If we indicate with cmin and cmax, respectively, the minimum and maximum normalized correlation coeffi cient values re- turned by the template matching function, the eye is inferred to be closed if

$\operatorname{Tmin} 1 \leq \operatorname{cmax} \leq \operatorname{Tmax} 1$ and

$\operatorname{Tmin} 2 \leq \mathrm{cmin} \leq \mathrm{T} \max 2$.

It is inferred to be open otherwise [1].

III. ADVANTAGES OF EYE PHONES 
The main advantages of using an eye tracking are:

i. $\quad$ Eye movement is faster than other input media that exists nowadays in mobile phones.

ii. $\quad$ Eye phones can make the miserable life of disabled person as better as normal one.

iii. Eye phones can be simply used by any users and no special learning is required.

iv. Eye phone can be useful in the matters of security which now a day is the biggest challenge.

$v$. Helpful for usability studies to understand users interact with their environments.

vi. It can also show determine as what specific information a user is searching for.

vii. Making video games and graphics more interesting.

viii. Potentially could provide new and more effective methods of computer-human interaction.

ix. $\quad$ It can be used even while walking.

\section{DISADVANTAGES OF EYE PHONE}

The main disadvantages of using an eye tracking are:

i. $\quad$ Eye detection and tracking could be possible go wrong by camera movements.

ii. More than one user can't access the phone at one time as in case of mobile phones.

iii. Continuously looking at the phones can greatly damage our iris and lenses.

iv. From the researches, it has been proved that eye phones have less accuracy in phones as compared to personal computers.

\section{APPLICATIONS OF EYE PHONE}

The major applications of using eye phones are:

i. The eye phone can be used to treat patients with eye diseases as eye phone can measure vision, takes pictures of the back of the eye etc. that can be shared with specialists anywhere in the world [5].

ii. Eye phone makes the gaming experience look more real as it adds virtual reality to it, thus making it more interesting.

iii. Eye phones can detect the drowsiness and distraction of the drivers and can be used to enhance their safety.

iv. $\quad$ Eye phone provides eye menus that can be used to access the phone easily when hands are busy or during driving etc.

\section{FUTURE SCOPE}

The work on the eye phone is still going on in many research and applied fields. The main works that must be carried out should focus on the following things:

i. $\quad$ More precise algorithms must be devised to detect the eye contour under various conditions.

ii. Improvement can be made in operating the eye phone under various movements and lightening conditions.

iii. Instead of using a fixed threshold policy, a learning approach can be used [6].

iv. Eye related measurements can contribute to the user's emotional and cognitive states [6].

$v$.Artificial algorithms and studies can be used to detect the natural behaviour and eye movements of the users.

\section{CONCLUSION}

The Eye phone is a hand free interfacing system that removes the gap between the real and the electronic world. It increases the human -phone interaction (HPI) and decreases the on board equipments and sensors. It just works on eye tracking and blink detection for operation the smart phone using the front camera. It is great importance in today's world where implicit commands become more useful and attractive that the explicit one. The security of our phone too will be greatly increased by the iris recognition which is quite reliable.

\section{ACKNOWLEGMENT}

We would like to thank our HOD Mrs. Yasmeen for providing us with useful and valuable comments on the study. This study would not have been possible without their support. All remaining errors, if any, are our responsibility. The usual disclaimer applies.

\section{REFERENCES}

1) https://www.researchgate.net/publication/221164350_EyePh one_Activating_Mobile_Phones_With_Your_Eyes

2) https://www.technologyreview.com/s/419033/eye-trackingformobile-control/

3) http://users.utcluj.ro/ tmarita/HCI/C7-8-extra/Eyeblink/2005012-blink-detection.pdf

4) https://www.tobiipro.com/fields-of-use/psychologyandneuroscience/visual-perception/

5) http://www.pharmaceuticaljournal.com/opinion/blogs/eyephoneapplication/11108551.blog

6) https://www.slideshare.net/Ar9393943693/eye-phoneseminarreport-661380 